\title{
Extracting the top-quark mass and Yukawa coupling from the threshold scan at CLIC
}

\author{
K. Nowak ${ }^{1) *}$, A.F. Żarnecki ${ }^{2) *}$ \\ ${ }^{*}$ Faculty of Physics, University of Warsaw, Poland.
}

\begin{abstract}
The Compact Linear Collider (CLIC) is a concept for a next-generation accelerator at CERN, colliding electrons and positrons at energies up to several TeV. One of the main goals at the initial CLIC running stage is the measurement of the top-quark mass and width in a scan of the beam energy through the pair production threshold. However, the shape of the threshold cross section depends not only on the top-quark mass but also on other model parameters as the top-quark width, top Yukawa coupling and the strong coupling constant.

We study the expected precision of the top-quark mass determination from the threshold scan. We use the most general fit approach with all relevant model parameters taken into account. In addition, we take the normalisation uncertainty into account as well as the expected constraints on the top Yukawa coupling and strong coupling constant from earlier experiments. We demonstrate that even in the most general approach the top-quark mass can be extracted with statistical precision of the order of 20 to $30 \mathrm{MeV}$. Additional improvement is expected if the running scenario is optimized. We also address the feasibility of the top Yukawa coupling determination from the threshold scan.
\end{abstract}

Published in Proc. SPIE 11176, Photonics Applications in Astronomy, Communications, Industry, and High-Energy Physics Experiments 2019, 1117648 (6 November 2019) https://doi.org/10.1117/12.2536861

(C) 2019 Society of Photo-Optical Instrumentation Engineers (SPIE). One print or electronic copy may be made for personal use only. Systematic reproduction and distribution, duplication of any material in this publication for a fee or for commercial purposes, and modification of the contents of the publication are prohibited.

This work was carried out in the framework of the CLICdp Collaboration

\footnotetext{
${ }^{1}$ k.nowak27@student.uw.edu.pl

2 zarnecki@fuw.edu.pl
} 


\title{
Extracting the top-quark mass and Yukawa coupling from the threshold scan at CLIC
}

\author{
Kacper Nowak ${ }^{\mathrm{a}}$ and Aleksander F. Żarnecki ${ }^{\mathrm{a}}$ \\ ${ }^{a}$ University of Warsaw, Faculty of Physics, ul. Pasteura 5, 02-093 Warsaw, Poland.
}

\begin{abstract}
The Compact Linear Collider (CLIC) is a concept for a next-generation accelerator at CERN, colliding electrons and positrons at energies up to several $\mathrm{TeV}$. One of the main goals at the initial CLIC running stage is the measurement of the top-quark mass and width in a scan of the beam energy through the pair production threshold. However, the shape of the threshold cross section depends not only on the top-quark mass but also on other model parameters as the top-quark width, top Yukawa coupling and the strong coupling constant.

We study the expected precision of the top-quark mass determination from the threshold scan. We use the most general fit approach with all relevant model parameters taken into account. In addition, we take the normalisation uncertainty into account as well as the expected constraints on the top Yukawa coupling and strong coupling constant from earlier experiments. We demonstrate that even in the most general approach the top-quark mass can be extracted with statistical precision of the order of 20 to $30 \mathrm{MeV}$. Additional improvement is expected if the running scenario is optimized. We also address the feasibility of the top Yukawa coupling determination from the threshold scan.
\end{abstract}

Keywords: CLIC project, top-quark physics, threshold scan, top-quark mass, top Yukawa coupling

\section{INTRODUCTION}

The research programs of the future linear $\mathrm{e}^{+} \mathrm{e}^{-}$colliders include a wide range of measurements related to top quark. Precise measurement of the top-quark mass is of particular importance, as it is essential for the understanding of the Higgs mechanism, electroweak symmetry breaking and for constraining many "new physics" scenarios. Both the International Linear Collider (ILC) ${ }^{1}$ and the Compact Linear Collider (CLIC) ${ }^{2,3}$ are expected to provide an opportunity to extract the top-quark mass from the measurement of the $t \bar{t}$ pair-production threshold around $\sqrt{s}=350 \mathrm{GeV}$. Detailed simulation studies were performed for ILC $^{4}$ and CLIC 5 showing that with cross section measurements at 10 different values of collision energy, each with $10 \mathrm{fb}^{-1}$ of data, a statistical accuracy of the order of $20 \mathrm{MeV}$ can be obtained on the top-quark mass. However, the shape of the threshold cross section is also sensitive to other model parameters: the top-quark width, its Yukawa coupling and the strong coupling constant. Uncertainties on these parameters together with systematic effects were shown to result in the total expected uncertainty on the top-quark mass of the order of $50 \mathrm{MeV} .{ }^{6}$ However, only the two-parameter fits to the threshold scan data were considered in this study.

Discussed in this contribution is the more general approach to the top-quark mass measurement at the threshold based on the simultaneous fit of all model parameters and the normalisation, taking also the possible constraints from earlier measurements into account. We examine constraints on the data normalisation, the strong coupling constant and the top Yukawa coupling required to extract the top-quark mass with the statistical precision of the order of $30 \mathrm{MeV}$. Further improvement of the measurement should be possible by optimizing the threshold scan scenario. ${ }^{7}$ Although the study was carried out in the framework of the CLICdp Collaboration presented results are more general and should apply to possible measurement at ILC as well.

Further author information: (Send correspondence to Kacper Nowak)

Kacper Nowak: E-mail: k.nowak27@student.uw.edu.pl

Aleksander F. Żarnecki: E-mail: zarnecki@fuw.edu.pl 


\section{REFERENCE SCENARIO}

The first stage of CLIC will allow an energy of $380 \mathrm{GeV}$ to be reached, giving access to most Higgs boson and top quark measurements. ${ }^{8}$ In the first CLIC running stage with $1 \mathrm{ab}^{-1}$ of total integrated luminosity, ${ }^{9}$ a dedicated scan of $100 \mathrm{fb}^{-1}$ is planned at the $t \bar{t}$ threshold. The baseline scenario of the threshold scan, considered for both ILC and CLIC, assumes running at 10 equidistant energy points taking $10 \mathrm{fb}^{-1}$ of data for each value of $\sqrt{s}$. Example of such a measurement is presented in Fig. 1. The solid curve represents the theoretical cross section predictions including the effects of the initial state radiation (ISR) and the CLIC luminosity spectra. For the presented study the "Low Charge" luminosity spectrum optimised for the threshold scan was used. ${ }^{6}$ Large sets of simulated measurement (pseudo-data) were generated taking into account the expected statistical uncertainties of the data points. The overall top-pair event reconstruction efficiency of $70.2 \%$, including the relevant branching fractions, was assumed, ${ }^{5}$ and the background contribution remaining after the event selection procedure corresponding to the cross section of $73 \mathrm{fb}$.

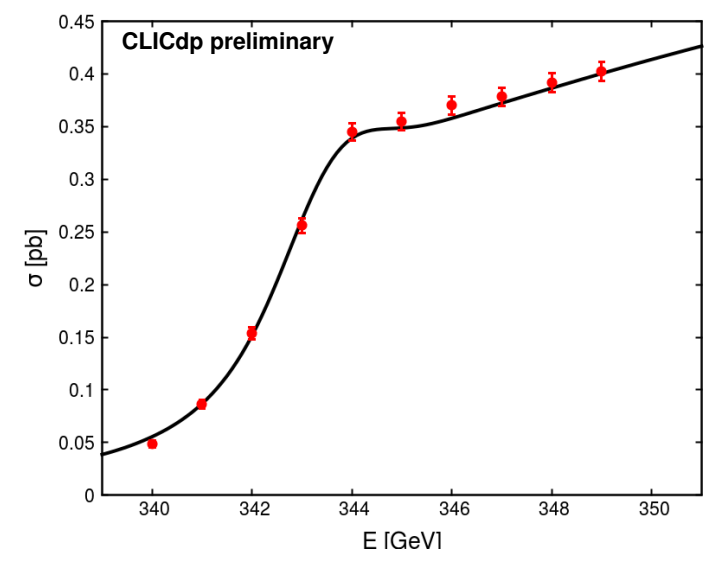

Figure 1. Reference scenario for the top-quark threshold scan. The error bars on the simulated data points show the expected statistical uncertainties of the cross section measurements assuming total integrated luminosity of $10 \mathrm{fb}^{-1} \mathrm{per}^{-}$ measurement and event selection efficiency of $70.2 \% .^{5}$

\section{FIT METHOD}

To calculate the cross section templates the program QQbar_threshold, ${ }^{10}$ taking into account NNNLO QCD calculations ${ }^{11}$ and other corrections ${ }^{12-14}$ was used. The templates were generated for different values of the top-quark mass ${ }^{*}, m_{t}$, top-quark width, $\Gamma_{t}$, top Yukawa coupling (relative to the SM value), $y_{t}$, and of the strong coupling constant, $\alpha_{s}$. Independent variations of all parameters were considered around the nominal values of $\tilde{m}_{t}=171.5 \mathrm{GeV}, \tilde{\Gamma}_{t}=1.37 \mathrm{GeV}, \tilde{y}_{t}=1$ and $\tilde{\alpha}_{s}\left(M_{Z}^{2}\right)=0.1185$. These templates are used to fit the parameter values to the simulated measurement results. For estimate of the theoretical uncertainties, we vary the renormalisation scale, $\mu$, used in the cross section calculations, between $60 \mathrm{GeV}$ and $120 \mathrm{GeV}$ (the nominal value is $\tilde{\mu}=80 \mathrm{GeV}$ ). We also assume $2 \%$ uncertainty on the expected background cross contribution. For a proper estimate of systematic effects, the corresponding variations were only applied in the pseudo-data generation and not in the fit.

For each generated pseudo-data set and each considered cross section template the $\chi^{2}$ value is calculated from the formula

$$
\chi^{2}(\vec{p}, \alpha)=\sum_{i}\left(\frac{m_{i}-\alpha \cdot \mu_{i}(\vec{p})}{\sigma_{i}}\right)^{2}+\left(\frac{\alpha-1}{\Delta}\right)^{2}+\sum_{j}\left(\frac{p_{j}-\tilde{p}_{j}}{\sigma_{p_{j}}}\right)^{2}
$$

where $m_{i}$ and $\sigma_{i}$ are the measured cross section values with their statistical uncertainties, $\mu_{i}(\vec{p})$ denotes the template cross section values for corresponding collision energies and given parameter set $\vec{p}=\left(m_{t}, \Gamma_{t}, \alpha_{s}, y_{t}^{2}\right)$,

${ }^{*}$ PS mass scheme ${ }^{15}$ is used for the top-quark mass definition. 
and $\alpha$ is the template normalisation factor. The second term in the $\chi^{2}$ formula corresponds to the normalisation constraint, where we consider different values of the relative normalisation uncertainty $\Delta$. The normalisation factor $\alpha$ can be evaluated for each template separately, by solving the minimisation condition $\frac{\partial \chi^{2}}{\partial \alpha}=0$. The third term represents possible constraints on the model parameters $p_{j}$ resulting from the earlier measurements with uncertainty $\sigma_{p_{j}}$. In this study, we consider possible external constraints for $\alpha_{s}$ and $y_{t}$ only.

The values of the top-quark mass and other parameters can then be extracted from the fit of the polynomial $\chi^{2}$ dependence on the components of $\vec{p}$

$$
\chi_{\alpha}^{2}(\vec{p})=\sum_{i=0}^{N} \sum_{j=0}^{N} A_{i, j} p_{i} p_{j}
$$

where $\chi_{\alpha}^{2}$ is the $\chi^{2}$ value minimised w.r.t. normalisation factor $\alpha, N$ is the number of considered model parameters and $i, j$ are parameter indexes $(i, j=1 \ldots N) . A_{i, j}$ is the symmetric matrix of coefficients of N-dimensional parabola. To simplify the formula, we define $p_{0} \equiv 1$ (the linear part is thus given by $2 A_{0, j} p_{j}$ and the constant part is $\left.A_{0,0}\right)$. The values of parameters $A_{0,0}, \ldots, A_{n, n}$ are found by solving a set of linear equations, when we use $\chi_{\alpha}^{2}$ values calculated for different parameter sets $\vec{p}$ as input. The fitted values of $A_{0,0}, \ldots, A_{n, n}$ are then used to extract the parameter values minimising the $\chi_{\alpha}^{2}(\vec{p})$, their expected uncertainties and correlation coefficients.

Results of the two parameter fit (top-quark mass and width) to about 4000 pseudo-data sets simulated for the reference threshold scan scenario (see Fig. 1) are presented in Fig. 2. The distribution of fit results is well described by the 2-D Gaussian profile; results of the profile fit to the data are indicated by the one, two and five sigma contours ${ }^{\dagger}$ in Fig. 2.

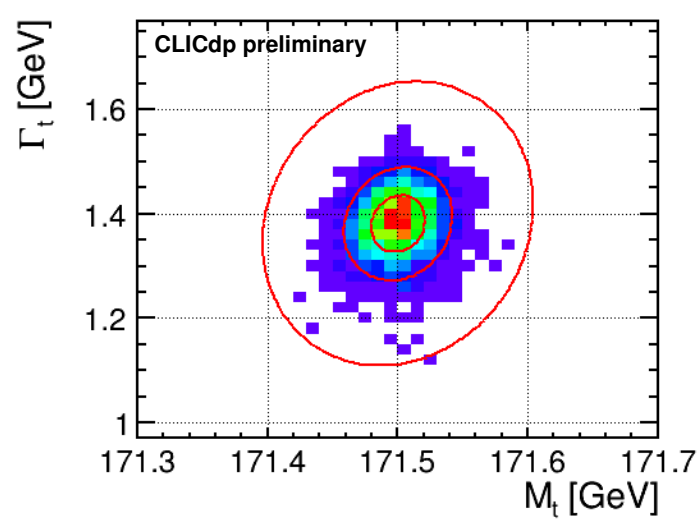

Figure 2. Results of the two-parameter fits to the top threshold scan, for sets of pseudo-data generated for nominal parameter values, $\tilde{m}_{t}=171.5 \mathrm{GeV}$ and $\tilde{\Gamma}_{t}=1.37 \mathrm{GeV}$. One, two and five sigma contours represent the 2-D gaussian distribution fitted to the presented results.

\section{VALIDATION}

To ensure that our fit algorithm gives proper results we repeated the fit procedure for sets of pseudo-data generated with non-standard templates. Shown in Fig. 3 are the distributions of the fitted top-quark mass from the two-parameter fit (2D) and the fitted top Yukawa coupling parameter from the four-parameter fit (4D), for the pseudo-data sets generated using cross-section templates with different values of these parameters. For both the top-quark mass and Yukawa coupling variations, fit results follow very closely the input parameter values.

Shown in Fig. 4 are the distributions of the top-quark mass uncertainty and the top-quark width uncertainty, resulting from the two-parameter fits to a large set of pseudo-data generated with a nominal template. These

\footnotetext{
${ }^{\dagger}$ Note that for the 2-D Gaussian distribution about $61 \%, 14 \%$ and $0.0004 \%$ of events are expected outside $1 \sigma, 2 \sigma$ and $5 \sigma$ contours, respectively.
} 

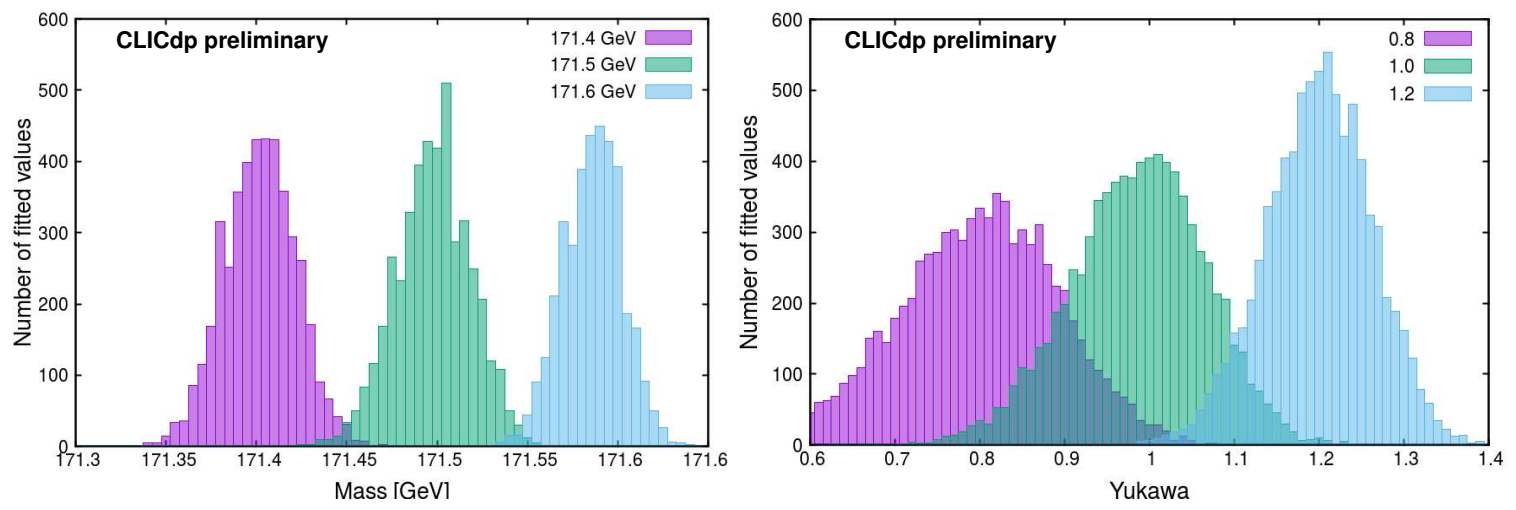

Figure 3. Histograms of fitted values for the top-quark mass (left) and top Yukawa coupling (right). Different histograms corresponds to the pseudo-data sets generated using cross-section templates with different values of the corresponding model parameter, as indicated in the plot.

uncertainties are calculated from the parameter covariance matrix, based on coefficients of the polynomial $\chi^{2}$ parameterisation described by Eq. 2. In the ideal case they should match the Gaussian widths of the parameter distributions. For the considered 2-D fit configuration (top-quark mass vs top-quark width, see Fig. 2), fits to the marginalized parameter distributions result in $\sigma_{m_{t}}=21 \mathrm{MeV}$ and $\sigma_{\Gamma_{t}}=54 \mathrm{MeV}$. These uncertainties are in a very good agreement with results of the previous study. ${ }^{6}$ The uncertainties estimated from the covariance matrix of the fit tend to be systematically lower, which can most likely be attributed to the assumed shape of the $\chi^{2}$ function, neglecting the possible higher-order terms. Still, we can conclude that our fit method reproduced nicely the input parameter values and gives realistic uncertainty estimates.
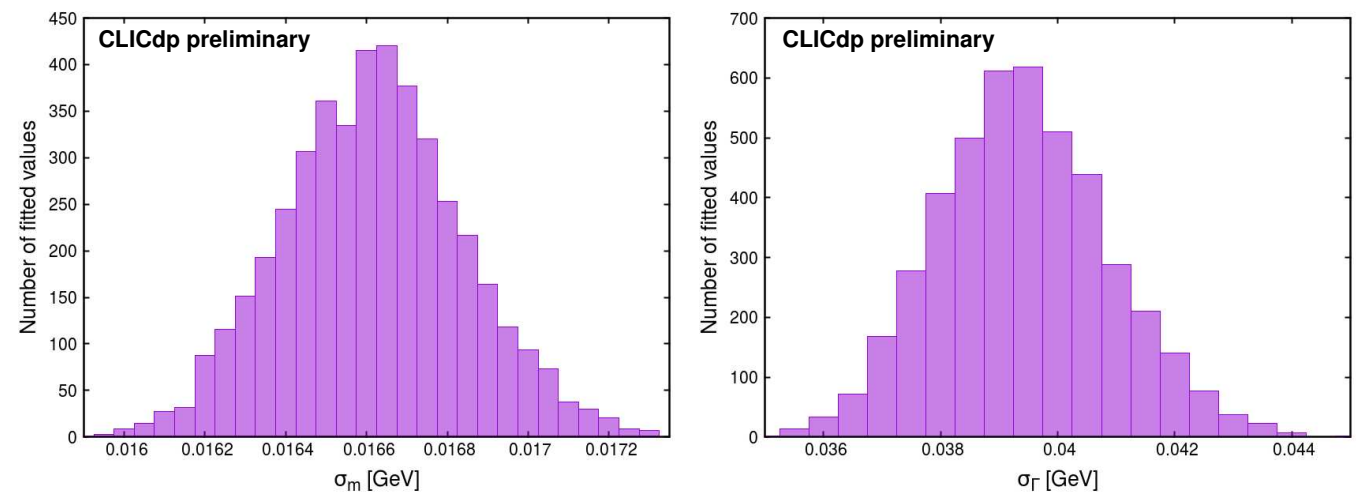

Figure 4. Distributions of the top-quark mass uncertainty (left) and the top-quark width uncertainty (right), from the two-parameter fits to a set of pseudo-data generated from the nominal template.

\section{RESULTS}

As mentioned above, all studies presented so far considered at most two-parameter fits to the threshold scan data. Our approach, thanks to its simple, semi-analytical form, allows us to perform fits with more free model parameters. Moreover, we can add additional constraints on the selected parameters to make the fit reflect the expected experimental situation better. The constraint that clearly influences the fit results is the assumed uncertainty on the data normalisation ${ }^{\ddagger}, \Delta$ (see Eq. 1). This influence is clearly visible in Fig. 5, which shows the expected statistical precision of the top-quark mass determination as a function of the normalisation uncertainty. Four different fit configurations are considered: two-dimensional fit of mass and width (2D), fit of mass, width

\footnotetext{
${ }^{\ddagger}$ This uncertainty also includes the possible overall normalisation uncertainty of the theoretical prediction.
} 


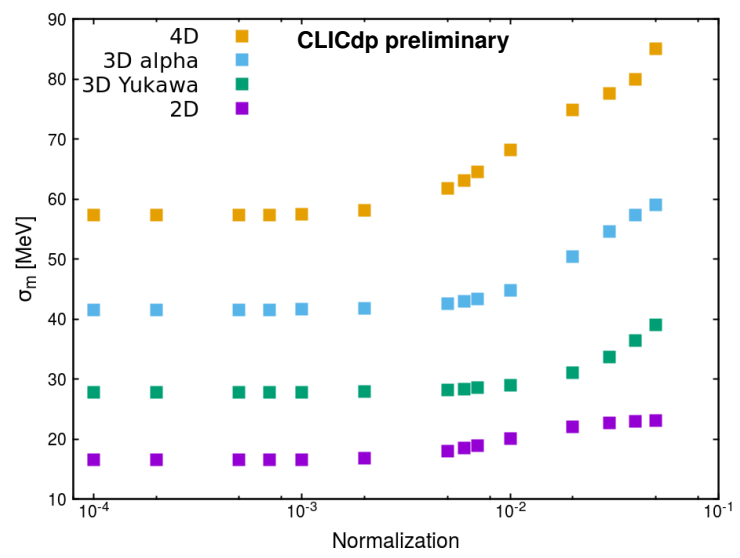

Figure 5. Statistical uncertainty on the top-quark mass as a function of the normalisation uncertainty. Four fit configurations are considered, see text for details.

and strong coupling (3D alpha), fit of mass, width and Yukawa coupling (3D Yukawa), and the simultaneous fit of the top-quark mass, width, strong coupling and Yukawa coupling (4D). Please note that normalisation is always fitted as an additional parameter, as described in Sec. 3. To allow for the most precise top-quark mass determination, the overall normalisation of the data (and theory) has to be known on sub-percent level. Also visible in Fig. 5 is the effect of adding more model parameters in the fit. While the statistical uncertainty on the top-quark mass below $20 \mathrm{MeV}$ can be easily achieved when we use the $2 \mathrm{D}$ fit procedure (i.e. mass and width fit only), the uncertainty increases significantly when other parameters (strong coupling or Yukawa coupling) are added to the fit. However, the strong coupling constant is not a "free" parameter, and we have to take into account that it can be constrained from other measurements. This is shown in Fig. 6, where the top-quark mass uncertainty from the 3D fit (with mass, width and strong coupling as free parameters, as well as normalisation) is plotted as a function of the strong coupling and normalisation uncertainties. To be able to extract the mass with $20 \mathrm{MeV}$ precision, the strong coupling constant has to be known to better than 0.0004 and uncertainty of 0.001 (current world average) would only allow to reach precision of the order of $30 \mathrm{MeV}$.

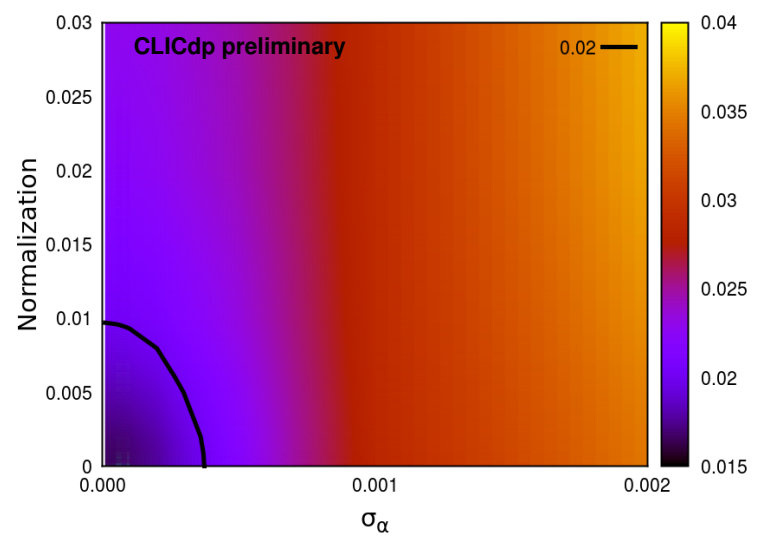

Figure 6. Expected statistical uncertainty on the top-quark mass as a function of the uncertainty of strong coupling constant and normalisation. Results from the fit of mass, width and the strong coupling (3D). Color maps are obtained using a linear interpolation algorithm.

The conclusions change significantly, if we include Yukawa coupling as an additional parameter in the fit. As shown in Fig. 7 (left), if no constraints are imposed on Yukawa coupling, the top-quark mass can not be extracted 

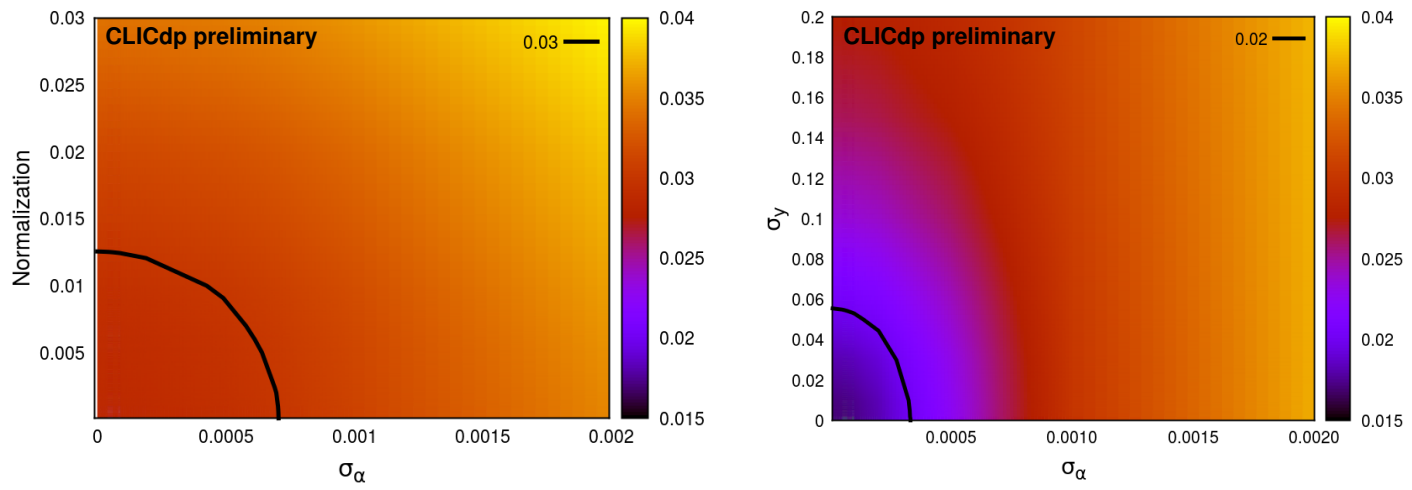

Figure 7. Expected statistical uncertainty on the top-quark mass from the fit involving all considered model parameters (4D). Left: as a function of the strong coupling constant and normalisation uncertainties, assuming no constraints on the Yukawa couplin. Right: as a function of the strong coupling constant and Yukawa coupling uncertainties, assuming normalisation uncertainty, $\Delta=10^{-3}$.

with precision better than $28 \mathrm{MeV}$, even for negligible uncertainties in normalisation and strong coupling (refer also to Fig. 5). To be able to extract the mass with precision of the order of $20 \mathrm{MeV}$, Yukawa coupling has to be constrained from other measurement to about $5 \%$ and the strong coupling constant has to be known to better than 0.0003 .

As the top-quark pair-production cross section depends on the top Yukawa coupling, the threshold scan data can also be used to constrain its value. We studied the statistical precision of the Yukawa coupling determination from the $4 \mathrm{D}$ fit as a function of the assumed normalisation and strong coupling uncertainties. Results presented in Fig. 8 indicate that contribution from the Yukawa coupling can be observed with high significance (above $5 \sigma$, i.e. with statistical precision below 0.2). Assuming the normalisation is known at per-mille level and the strong coupling is independently measured to better than 0.0003, statistical precision on the Yukawa coupling of the order of $10 \%$ should be feasible.

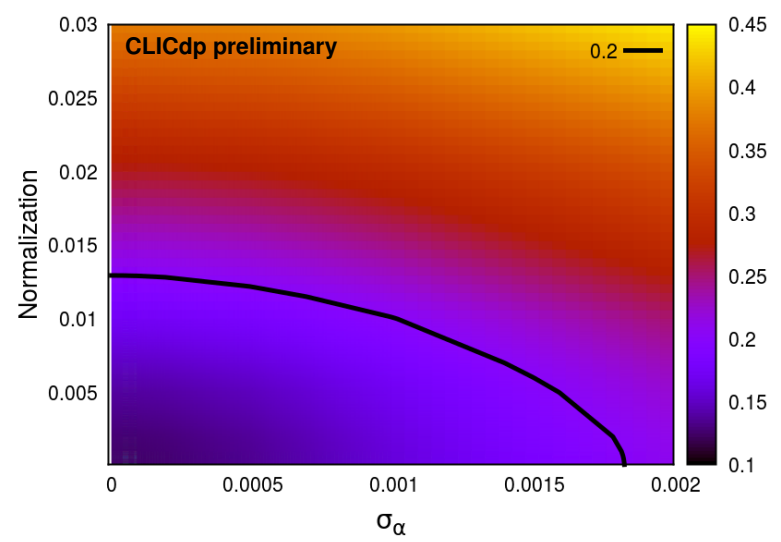

Figure 8. Expected statistical uncertainty on the top Yukawa coupling from the fit involving all considered model parameters $(4 \mathrm{D})$, as a function of the strong coupling constant and normalisation uncertainties. The black line indicates the parameter range where we can expect $5 \sigma$ significance. 


\section{SYSTEMATIC EFFECTS}

The systematic uncertainties of the threshold cross section measurements are likely to limit the ultimate precision of the top-quark mass determination. Various sources of uncertainties have been investigated ${ }^{6}$ and the combined systematic uncertainties on the top-quark are expected to be in the range $30 \mathrm{MeV}$ to $50 \mathrm{MeV}$. Here, we estimate the size of systematic effects in the top Yukawa coupling determination from the threshold scan. We repeated the 4-D fit procedure for pseudo-data sets generated with different systematic variations, as discussed in Sec. 3. Results are presented in Fig. 9. Variation of the renormalisation scale, $\mu$, has a negligible effect on the Yukawa coupling fit results. However, the assumed uncertainty on the background contribution of $2 \%$ results in about $15 \%$ systematic uncertainty in the determination of the top Yukawa coupling. One can conclude that the systematic effects will be important for constraining the top Yukawa coupling from the threshold scan and should be studied in more detail.
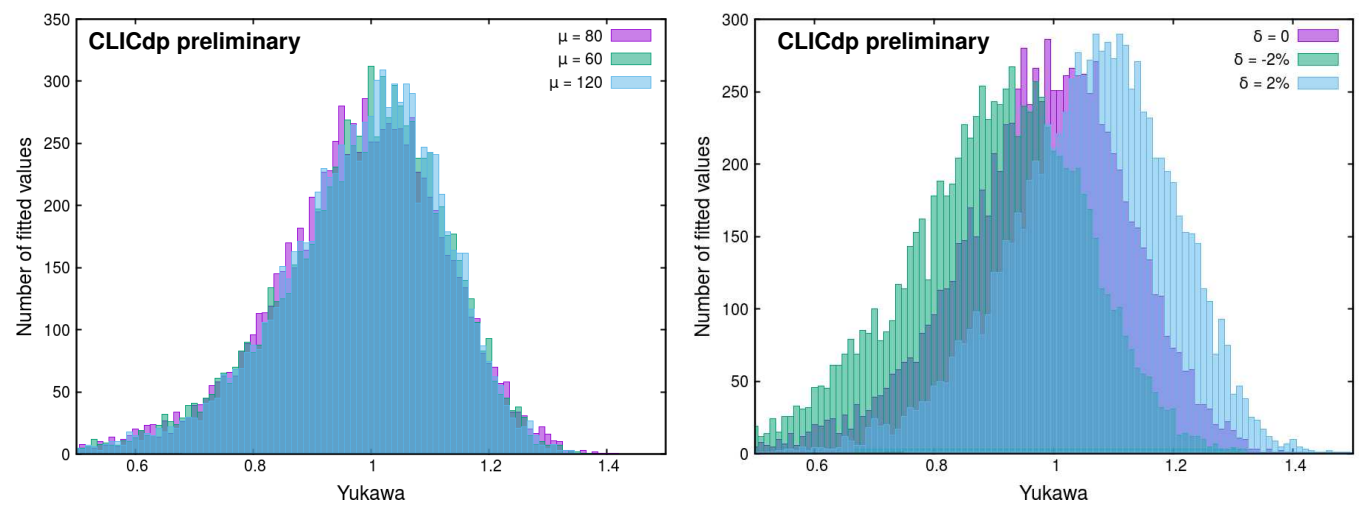

Figure 9. Histograms of the fitted top Yukawa coupling from the fits involving all considered model parameters (4D). Different histograms correspond to the pseudo-data sets generated with systematic variations of the renormalisation scale $\mu$ (left) and of the background level (right), as indicated in the plot.

\section{CONCLUSIONS}

We presented the general approach to the analysis of the top-quark pair-production threshold scan results, which is used to extend the results of the recent CLICdp study. ${ }^{6}$ A simultaneous fit of all relevant model parameters and normalisation is performed, taking into account possible constraints from other measurements. Results based on large sets of simulated measurements show that the statistical precision of top-quark mass measurement of the order of $30 \mathrm{MeV}$ can be easily obtained, while reducing this uncertainty further is only possible, if uncertainties on the normalisation, strong coupling constant and the Yukawa coupling are sufficiently low. In particular, to reduce the statistical uncertainty to the level of $20 \mathrm{MeV}$, the Yukawa coupling needs to be known to about $5 \%$.

Top threshold scan data can be also used to constrain the top Yukawa coupling. Statistical precision of the order of $10 \%$ can be obtained, provided the normalisation is known at per-mille level and the strong coupling to better than 0.0003 . If this is the case, determination of the Yukawa coupling from the threshold scan data will be limited by theoretical uncertainties and systematic effects.

\section{ACKNOWLEDGMENTS}

This work was carried out in the framework of the CLICdp Collaboration. We are grateful to collaboration members for fruitful discussions and comments received to the manuscript. 


\section{REFERENCES}

[1] "The International Linear Collider Technical Design Report - Volume 2: Physics," (2013). ILC-Report-2013040, arXiv:1306.6352.

[2] Linssen, L. et al., eds., [Physics and Detectors at CLIC: CLIC Conceptual Design Report], CERN (2012). CERN-2012-003.

[3] Charles, T. K. et al., "The Compact Linear Collider (CLIC) - 2018 Summary Report," CERN Yellow Rep. Monogr. 1802, 1-98 (2018).

[4] Horiguchi, T., Ishikawa, A., Suehara, T., Fujii, K., Sumino, Y., Kiyo, Y., and Yamamoto, H., "Study of top quark pair production near threshold at the ILC." arXiv:1310.0563 (2013).

[5] Seidel, K., Simon, F., Tesar, M., and Poss, S., "Top quark mass measurements at and above threshold at CLIC," Eur. Phys. J. C73, 2530 (2013).

[6] Abramowicz, H. et al., "Top-Quark Physics at the CLIC Electron-Positron Linear Collider." arXiv:1807.02441 (Submitted to JHEP) (2018).

[7] Nowak, K. and Zarnecki, A. F., "Prospects for improving top-quark mass measurement precision at future e+e- colliders," Proc.SPIE 10808, 10808 - 10808 - 8 (2018).

[8] Boland, M. J. et al., "Updated baseline for a staged Compact Linear Collider." CERN-2016-004, arXiv: 1608.07537 (2016).

[9] Robson, A. and Roloff, P., "Updated CLIC luminosity staging baseline and Higgs coupling prospects." CLICdp-Note-2018-002, arXiv:1812.01644 (2018).

[10] Beneke, M., Kiyo, Y., Maier, A., and Piclum, J., "Near-threshold production of heavy quarks with QQbar_threshold," Comput. Phys. Commun. 209, 96-115 (2016).

[11] Beneke, M., Kiyo, Y., Marquard, P., Penin, A., Piclum, J., and Steinhauser, M., "Next-to-Next-to-Next-toLeading Order QCD Prediction for the Top Antitop $S$-Wave Pair Production Cross Section Near Threshold in $e^{+} e^{-}$Annihilation," Phys. Rev. Lett. 115(19), 192001 (2015).

[12] Beneke, M., Maier, A., Piclum, J., and Rauh, T., "Higgs effects in top anti-top production near threshold in $e^{+} e^{-}$annihilation," Nucl. Phys. B899, 180-193 (2015).

[13] Beneke, M., Piclum, J., and Rauh, T., "P-wave contribution to third-order top-quark pair production near threshold," Nucl. Phys. B880, 414-434 (2014).

[14] Beneke, M., Jantzen, B., and Ruiz-Femenia, P., "Electroweak non-resonant NLO corrections to $e^{+} e^{-} \rightarrow$ $W^{+} W^{-} b \bar{b}$ in the $t \bar{t}$ resonance region," Nucl. Phys. B840, 186-213 (2010).

[15] Beneke, M., "A Quark mass definition adequate for threshold problems," Phys. Lett. B434, 115-125 (1998). 